\title{
Nonequilibrium Interfacial Tension in Simple and Complex Fluids
}

\author{
Domenico Truzzolillo, ${ }^{1, *}$ Serge Mora, ${ }^{2}$ Christelle Dupas, ${ }^{1}$ and Luca Cipelletti ${ }^{1}$ \\ ${ }^{1}$ Laboratoire Charles Coulomb (L2C), UMR 5221 CNRS-Université de Montpellier, \\ F-34095 Montpellier, France \\ ${ }^{2}$ Laboratoire de Mécanique et de Génie Civil, UMR 5508 CNRS et Université de Montpellier 2, \\ Place E. Bataillon F-34095 Montpellier cedex 5, France \\ (Received 16 June 2016; revised manuscript received 3 September 2016; published 19 December 2016)

\begin{abstract}
Interfacial tension between immiscible phases is a well-known phenomenon, which manifests itself in everyday life, from the shape of droplets and foam bubbles to the capillary rise of sap in plants or the locomotion of insects on a water surface. More than a century ago, Korteweg generalized this notion by arguing that stresses at the interface between two miscible fluids act transiently as an effective, nonequilibrium interfacial tension, before homogenization is eventually reached. In spite of its relevance in fields as diverse as geosciences, polymer physics, multiphase flows, and fluid removal, experiments and theoretical works on the interfacial tension of miscible systems are still scarce, and mostly restricted to molecular fluids. This leaves crucial questions unanswered, concerning the very existence of the effective interfacial tension, its stabilizing or destabilizing character, and its dependence on the fluid's composition and concentration gradients. We present an extensive set of measurements on miscible complex fluids that demonstrate the existence and the stabilizing character of the effective interfacial tension, unveil new regimes beyond Korteweg's predictions, and quantify its dependence on the nature of the fluids and the composition gradient at the interface. We introduce a simple yet general model that rationalizes nonequilibrium interfacial stresses to arbitrary mixtures, beyond Korteweg's small gradient regime, and show that the model captures remarkably well both our new measurements and literature data on molecular and polymer fluids. Finally, we briefly discuss the relevance of our model to a variety of interface-driven problems, from phase separation to fracture, which are not adequately captured by current approaches based on the assumption of small gradients.
\end{abstract}

DOI: 10.1103/PhysRevX.6.041057

Subject Areas: Fluid Dynamics, Soft Matter

\section{INTRODUCTION}

The spherical shape of soap bubbles and liquid droplets, the capillary forces responsible, e.g., for the sap rise in trees, the locomotion of water-strider insects, and the fractionation of a liquid jet exploited in the spray or automotive industry are only a few among the very numerous manifestations of the interfacial tension between immiscible phases. Given its pervasiveness, it is not surprising that interfacial tension has been the subject of extensive investigations for more than three centuries [1-4], from the nature of the interface between immiscible fluids to recent work on the shape of soft, viscoelastic solids $[5,6]$. The importance of interfacial phenomena has been further highlighted by the very rapid emergence of microfluidics and nanofluidics, where interface dynamics strongly affects the transport and response of fluids [7,8].

From a mechanical point of view, interfacial tension arises from the difference between the normal and

*domenico.truzzolillo@umontpellier.fr

Published by the American Physical Society under the terms of the Creative Commons Attribution 3.0 License. Further distribution of this work must maintain attribution to the author(s) and the published article's title, journal citation, and DOI. tangential components of the stress tensor in the proximity of the interface separating two phases [3]. More than a century ago, Korteweg was the first to propose that similar stresses may also exist, albeit transiently, when two miscible fluids are brought in contact $[9,10]$. The idea that interfacial stresses shape the transition region between miscible liquids, as they do for immiscible fluids, goes back at least to a 1871 report by Bosscha, cited in the 1901 paper by Korteweg [9]. In order to put these observations on a firm theoretical ground, Korteweg introduced in the Navier-Stokes equations a tangential stress term arising from the composition gradient at the interface. This term mimics the effect of an interfacial tension, which is referred to as the effective interfacial tension $\Gamma_{e}$. Assuming that the concentration gradient is small and linear and invoking local equilibrium leads to the so-called square gradient model [10-12], which predicts a quadratic dependence of $\Gamma_{e}$ on the compositional mismatch $\Delta \varphi$ between the bulk phases:

$$
\Gamma_{e}=\kappa \frac{\Delta \varphi^{2}}{\delta} .
$$

Here, $\kappa$ is the Korteweg or square gradient parameter, $\delta$ the thickness of the interface, and $\Delta \varphi$ is expressed in terms of a 
difference in volume fraction, e.g., between a solute and the solvent for molecular fluid [13-16], or between polymer or colloidal suspensions and their solvent for complex fluids $[17,18]$.

Clearly, the interfacial tension described by Eq. (1) can only exist transiently, before diffusion or other mixing mechanisms smear out the interface leading to a single, homogeneous phase. Notwithstanding this transient, nonequilibrium character, the effective interfacial tension between miscible fluids plays a key role in a wide range of research fields of both academic and practical interest [19], from geosciences (e.g., in mantle convection, magma fragmentation, and the dynamics of Earth's core $[20,21]$ ) to hydrology [22], oil recovery [10], filtration and flow in porous media (e.g., in a chromatography column [23]), fluid removal [24], aquifer and soil remediation [25,26], and the modeling of hydrodynamic instabilities, e.g., in Rayleigh-Taylor [27] and Hele-Shaw [28,29] flows. Very recently, convection induced by Korteweg stresses has been proposed as a new mechanism for self-propulsion of droplets [30] and vesicles [31], demonstrating artificial chemotaxis and opening new scenarios in active matter and drug delivery systems. We may thus state quite generally that effective interfacial tension plays an important role in all miscible multifluid systems and at all length scales.

In spite of its importance, our understanding of the effective interfacial tension is still limited and several fundamental questions are not settled yet. The very existence of an interfacial tension between miscible fluids is debated. The classical theory of capillarity predicts that the interfacial tension vanishes in the limit of fully miscible fluids. [32]. Simulations [33] and experiments [34,35] probing the Saffman-Taylor instability led to the conclusions that no interfacial tension exists between miscible fluids; in particular, the observation of a fractal-like interface between two miscible fluids was attributed to a vanishing interfacial energy cost, i.e., to $\Gamma_{e}=0[34,35]$. This has to be contrasted with experiments and simulations on miscible fluids probing capillary waves [14,15,36], the shape of drops and menisci $[13,16,17,37,38]$, and that of patterns in hydrodynamic instabilities [18,27-29,39-41], which all claimed the existence of an effective interfacial tension. The sign of $\Gamma_{e}$ is also debated. This has profound implications on the evolution and stability of the interface, since a positive (negative) sign corresponds to a stabilizing (destabilizing) effect against the creation of additional interfacial area. While experiments generally suggest $\Gamma_{e}>0$, a recent dynamic density-functional theory study [42] reports a negative interfacial tension in complex fluids composed of repulsive particles, in the short-time limit. A similar result has been obtained in calculations of the interfacial tension between dense and dilute phases of active Brownian particles [43] and in molecular dynamics simulations measuring the square gradient parameter of water and olefines [44].
On the theoretical side, a comprehensive description of out-of-equilibrium interfacial phenomena is still missing. The only available theory, Korteweg's square gradient model [Eq. (1)], is restricted to small gradients of the compositional mismatch. No a priori arguments are available to quantitatively asses whether Korteweg's law would be adequate to model a given system. Moreover, deviations from Korteweg's $\Delta \varphi^{2}$ scaling have been clearly observed in molecular [13] and complex fluids alike [16,41], thus demonstrating the need for a more general theory. Even for those systems where Korteweg's law correctly captures the $\Delta \varphi$ scaling of $\Gamma_{e}$, no predictions are available for the magnitude of the square gradient coefficient $\kappa$, with the exception of microgel colloidal particles [18]. This limits the usefulness of Eq. (1) and prevents the interface thickness - an important parameter experimentally difficult to access directly - from being inferred from a measurement of $\Gamma_{e}$ versus $\Delta \varphi$.

More broadly, square gradient-based formulas are at the core of the density-functional theory [45] and the "phase field" method [46], two powerful techniques for the description of inhomogeneous systems, from binary mixtures of phase separating fluids to metallic alloys [47], crystal growth and defects [48,49], and fractures [50]. While a square-gradient approach is usually adequate for the description of systems with smeared interfaces, there is a need for more-refined approaches to satisfactorily describe sharp interfaces within the density-functional theory or phase field method [44,47-51]. Thus, any progress in modeling the effective interfacial tension beyond Korteweg's square-gradient formalism is likely to also have an impact on the broader problem of modeling sharp interfaces in multiphase systems.

Here, we investigate the effective interfacial tension with both experiments and theoretical modeling. The experiments leverage on the unique features of complex fluids such as polymer and colloidal suspensions: more accessible time and length scales and slower diffusion-and thus longerlived interfaces - as compared to molecular fluids, and the possibility of systematically varying the particle structure and interparticle interactions. Experimentally, we establish several key results. We show that a nonvanishing, positive interfacial tension exists between a variety of complex fluids and their solvent. We uncover that both the magnitude and the concentration dependence of $\Gamma_{e}$ depend dramatically on the nature of the fluids. For long polymer chains, $\Gamma_{e}$ is consistent with Korteweg's quadratic scaling. By contrast, for suspensions of compact colloidal particles $\Gamma_{e}$ exhibits a striking, exponential-like increase with $\Delta \varphi$. For these systems, $\Gamma_{e}$ varies over 5 decades for mild changes in concentration, reaching values as high as tens of $\mathrm{mN} \mathrm{m}^{-1}$, close to the interfacial tension of molecular fluids and orders of magnitude larger than the typical interfacial tension of equilibrium colloidal phases. Short polymer chains display a behavior intermediate between that of long polymers and 
compact particles: as the chain length decreases, the growth of $\Gamma_{e}$ with concentration becomes increasingly steeper, departing from Korteweg's quadratic scaling. As a first step towards rationalizing these results and previous literature data for complex fluids and molecular systems, we explicitly calculate Korteweg's constant for polymer solutions and molecular mixtures. We then go beyond Korteweg's small gradient approximation and introduce a simple yet general model applicable to systems with arbitrarily large composition mismatches. The model quantitatively accounts for the full spectrum of the observed behaviors, with adjustable parameters-the interface thickness, an additional length scale, and a free-energy density scale-consistent with estimates based on simple physical arguments.

Our experiments and theoretical modeling provide a unified framework for understanding and tailoring offequilibrium interfacial stresses, with implications from geosciences to microfluidic and active matter. More generally, the model proposed here provides a compact yet powerful description of the interface contribution to the (free) energy of a multiphase system, for arbitrary concentration gradients. We thus expect it to be of use in the description of systems with sharp interfaces, namely within density-functional theory or phase field approaches.

\section{EFFECTIVE INTERFACIAL TENSION MEASUREMENTS}

\section{A. Setup}

We measure $\Gamma_{e}$ by analyzing the patterns formed when injecting the solvent in a colloid or polymer suspension, confined between two closely spaced, parallel plates (HeleShaw geometry). The interface between the two fluids develops a distinctive instability, termed viscous fingering or Saffman-Taylor instability, resulting from the competition between the viscosity contrast between the fluids, which tends to destabilize the interface, and interfacial tension, which tends to minimize the surface fluctuations. The Hele-Shaw cell consists of two square glass plates of side $L$ separated by four spacers of thickness $b$, in the range $0.25 \leq b \leq 1.00 \mathrm{~mm}$. To optimize the visualization of the fingers, both $L$ and $b$ are chosen according to the typical wavelength of the instability. For very low interfacial tensions, we use $L=50 \mathrm{~mm}$ and $b=1 \mathrm{~mm}$ in order to minimize the number of fingers, to avoid nonlinear effects occurring after the onset of the instability, such as tip splitting, and to enlarge the field of view. For large $\Gamma_{e}$, a smaller cell $(L=25 \mathrm{~mm})$ and a thinner gap $(b=0.25 \mathrm{~mm})$ are used, to favor the destabilization of the interface in order to clearly observe the instability. For some selected samples, we check the robustness of our method by performing experiments at different gaps, finding no dependence of $\Gamma_{e}$ on $b$.

The cell is initially filled with the more viscous fluid (the suspension), with viscosity $\eta_{2}$, and left to equilibrate for
$180 \mathrm{~s}$. The less viscous fluid is then injected through a hole of radius $r_{0}=0.5 \mathrm{~mm}$ in the center of the top plate. For all experiments, we use the solvent of the suspensions with $0.5 \% \mathrm{w} / \mathrm{w}$ of methylene blue as the less viscous fluid, with viscosity $\eta_{1}=1.011 \mathrm{mPas}$ (water) for most samples, or $\eta_{1}=15.570 \mathrm{mPa}$ s (water-glycerol mixture) for some colloidal suspensions. The injected volume per unit time $\dot{V}$ is controlled via a syringe pump. A fast CMOS camera (Phantom v7.3 by Vision Research) run at 100 to 3000 frames per second is used to record movies during the injection, by imaging the sample through the bottom plate.

\section{B. Analysis of the viscous fingering patterns}

We briefly recall the method introduced in Ref. [18] and used here to obtain $\Gamma_{e}$ from an analysis of the fingering instability. In the framework of a linear evolution theory, the instability is conveniently described by decomposing the interface profile in Fourier modes. In experiments, the number of lobes, or fingers, observed at the onset of the instability corresponds to $n_{A}$, the Fourier mode of the instability with maximum amplitude [18,29]. For data analysis, the relation between $n_{A}$ and the desired interfacial tension $\Gamma_{e}$ is conveniently expressed by introducing the finger function $K$ [18]:

$$
K\left(n_{A}, r\right)=\frac{b\left(16.85 n_{A}^{2}-1\right)}{4 r r_{0}\left[\eta_{2}\left(\dot{\gamma}_{r}\right)-\eta_{1}\right]}=\frac{1}{\Gamma_{e}} \dot{\gamma}_{I},
$$

where $r=\left[r_{0}^{2}+\dot{V} t /(\pi b)\right]^{0.5}$ is the time-dependent average radius of the interface, $\Gamma_{e}$ the interfacial tension between the two fluids, $\dot{\gamma}_{I}=3 \dot{V}\left(2 \pi r_{0} b^{2}\right)^{-1}$ the shear rate at the injection hole, and $\eta_{2}\left(\dot{\gamma}_{r}\right)$ the shear rate-dependent viscosity $[18,52,53]$, with $\dot{\gamma}_{r}=\left(4 r_{0} \dot{\gamma}_{I} / r\right)$ the shear rate calculated at a distance $r$ from the injecting hole, where the onset of the instability is observed. The shear-rate-dependent viscosity $\eta_{2}$ is measured independently by standard rheometry [54]. Operationally, for a sample at a given volume fraction $\varphi$, several experiments are performed at different injection rates and $\Gamma_{e}$ is obtained by fitting Eq. (2) to the experimental $K$ versus $\dot{\gamma}_{I}$ curve. The injection rate $\dot{\gamma}_{I}$ is kept high enough and the experiment duration short enough (from a few $\mathrm{ms}$ to a few hundreds of ms depending on the fluid viscosity) to make diffusion-driven mixing between the two fluids negligible [55]. All experiments are performed in the range of Reynolds number $10^{-2} \lesssim \operatorname{Re} \lesssim 10$, where Darcy's law [56] and the linear stability analysis of the Saffman-Taylor instability are applicable.

\section{Samples}

We study suspensions of colloidal particles $(C P)$ and solutions of linear polymers (LP) (see Table I) of different chemistry, size, and molecular weight, at volume fractions $\varphi$ ranging from $2.8 \times 10^{-3}$ to 0.633 , depending on the sample [57]. For polymers, $\varphi$ is the volume fraction 
TABLE I. Molecular mass $M_{w}$, degree of polymerization $N$, hydrodynamic radius $R_{h}$, and Flory parameter $\chi$ for the various polymers and colloidal particles used in the viscous fingering experiments. The last column reports the overlap concentration $\varphi^{*}$, for the polymers, and the packing fraction $\varphi_{c}$ where the zeroshear viscosity appears to diverge, for the colloids.

\begin{tabular}{lccccc}
\hline \hline Code & $M_{w}(\mathrm{Da})$ & $N$ & $R_{h}(\mathrm{~nm})$ & $\chi^{\mathrm{a}}$ & $\varphi^{* \mathrm{~b}}, \varphi_{c}{ }^{\mathrm{c}}$ \\
\hline LP-PSS2260 & $2.6 \times 10^{6}$ & 12942 & 110 & 1.10 & 0.0061 \\
LP-PSS666 & $6.7 \times 10^{5}$ & 3233 & 79 & 1.10 & 0.0064 \\
LP-PEG100 & $1.0 \times 10^{5}$ & 1611 & 8 & 0.45 & 0.0446 \\
LP-PEG35 & $3.5 \times 10^{4}$ & 564 & 4.5 & 0.45 & 0.0877 \\
LP-PEG2 & $2.0 \times 10^{3}$ & 32 & 1 & 0.45 & 0.1354 \\
MG-PNIPAm & $5.8 \times 10^{8}$ & $4.6 \times 10^{6}$ & 165 & 0.375 & \\
CP-Ludox & & & 18 & & 0.38 \\
CP-Pema & & & 122 & & 0.55 \\
CP-silica & & & 697 & & 0.62 \\
\hline \hline
\end{tabular}

${ }^{\mathrm{a}}$ All values are for polymers dissolved in water [63-65]. For the PEG and PNIPAm samples, we use the average of the values reported in literature.

${ }^{\mathrm{b}}$ The overlap concentration is calculated according to $\varphi^{*}=0.64 M_{w} /\left(\frac{4}{3} \pi R_{h}^{3} N_{A} \rho_{p}\right)$, where $N_{A}$ is Avogadro's number and $\rho_{p}$ the polymer mass density.

${ }^{c} \varphi_{c}$ is the volume fraction at which the zero-shear viscosity of the suspension apparently diverges, as obtained by fitting $\eta(\varphi)$ to the Krieger-Dougherty equation [66], $\eta \propto\left(1-\varphi / \varphi_{c}\right)^{-2}$.

occupied by the monomers, calculated from the mass fraction used to prepare the sample and the known polymer mass density. For colloids, $\varphi$ is obtained from the particle density and the mass fraction measured by drying a small amount of suspension. Polyethylene glycol (LP-PEG) and linear polystyrene sulfonate (LP-PSS) are purchased from Sigma-Aldrich and suspended in pure water $\left(\eta_{1}=1 \mathrm{mPa} \mathrm{s}\right)$ without any further purification or addition. For the sake of completeness, we also include the data of Ref. [18] on water-based microgels formed by cross-linked poly(Nisopropylacrylamide) (MG-PNIPAm), synthesized according to the protocol of Ref. [60]. Colloidal particles are made of silica ( $C P$-Ludox, Ludox-TM 50 purchased from Sigma-Aldrich and used as is; $C P$-silica, silica particles synthesized according to Ref. [61]) or poly(ethyl methacrylate) (CP-Pema, synthesized according to Ref. [62]). Preliminary data for $C P$-Ludox at the higher volume fractions were reported in Ref. [41]. For most of the $C P$ samples, the solvent is water, to which salt is added to partially screen the Coulombic repulsion due to surface charges: we use $50 \mathrm{mmol} \mathrm{KCl}$ for $C P$-Ludox and $C P$-silica, and $250 \mathrm{mmol} \mathrm{NaCl}$ for $C P$-Pema. For the $C P$-Ludox samples with $\varphi \leq 0.25$, the viscosity contrast between the water-based suspensions and water is too low and particle diffusion too fast for the viscous fingering instability to be observable. We thus use a more viscous mixture of water and glycerol (2/3 water/glycerol v/v, viscosity $\eta_{1}=15.57 \mathrm{mPa}$ ) as a solvent, with the same salt content as in the water-based samples. Details on all samples are reported in Table I. Note that for $C P$-silica the critical packing fraction $\varphi_{c}$ where the suspension viscosity seemingly diverges is close to $\varphi_{\text {rcp }} \approx 0.64$, the volume fraction of randomly close-packed spheres. This indicates that interparticle interactions are nearly hard-sphere-like. For $C P$-Pema and $C P$-Ludox, by contrast, $\varphi_{c}$ is substantially lower, due to long-range electrostatic repulsions.

In addition to our data, we also analyze literature data obtained by spinning drop tensiometry for dodecyl acrylate-poly(dodecyl acrylate) mixtures [17] (DA-PDA, the solvent is pure DA) and water-glycerol mixtures [13] (the solvent is pure water). The relevant parameters for these systems are summarized in Table II. For consistency with the other sets of data, we express concentration as the volume fraction $\varphi$ of PDA and of glycerol, for the data of Refs. [17] and [13], respectively.

\section{Experimental results}

For all polymer and colloidal suspensions displaced by their solvent, the interface exhibits a distinctive finger pattern, qualitatively similar to that obtained for immiscible fluids with positive interfacial tension: a $\dot{\gamma}$-dependent number of fingers [54] is systematically observed. This rules out the possibility that the interfacial tension be null or negative: in fact, $\Gamma_{e} \leq 0$ would lead to the "ultraviolet catastrophe" of the Saffman-Taylor instability $[69,70]$. The dispersion relation would not show any maxima, the dominant wavelength of the instability would be independent of $\dot{\gamma}_{I}$, and it would be limited only by the shortest length scale of the system, the gap $b$ [33]. By contrast, our data are very well fitted by the proportionality law $K=\dot{\gamma}_{I} / \Gamma_{e}$ [54], thereby demonstrating that the interfacial tension is positive and that it can be reliably extracted from the analysis of the Saffman-Taylor instability via Eq. (2).

We investigate systematically the $\varphi$ and sample dependence of $\Gamma_{e}$ and summarize our results and previous literature data in Fig. 1. Note that for selected samples the gap has been varied and the same value of $\Gamma_{e}$ (within experimental uncertainty) has been found regardless of $b$,

TABLE II. Sample parameters for the spinning drop tensiometry experiments of Refs. [13,17].

\begin{tabular}{lcccc}
\hline \hline & $M_{w}(\mathrm{Da})$ & $N$ & $R_{h}, a(\mathrm{~nm})^{\mathrm{a}}$ & $\chi$ \\
\hline PDA & 25000 & 104 & $4^{\mathrm{b}}$ & $0^{\mathrm{c}}$ \\
Glycerol & & & 0.4 & $-0.96^{\mathrm{d}}$ \\
\hline \hline
\end{tabular}

${ }^{\mathrm{a}}$ For the water-glycerol mixtures, $a$ is the radius of the glycerol molecule.

${ }^{\mathrm{b}} R_{h}$ has been estimated from the radius of Poly(dodecyl methacrilate) (PDMA) in good solvent [67], by rescaling the size of PDMA in order to take into account the difference in molar mass between PDA and PDMA.

${ }^{c} \chi=0$ if the solvent is composed by the same monomers that form the polymer [68].

${ }^{\mathrm{d}}$ Estimated from the enthalpy of mixing of water-glycerol (See Supplemental Material [54]). 


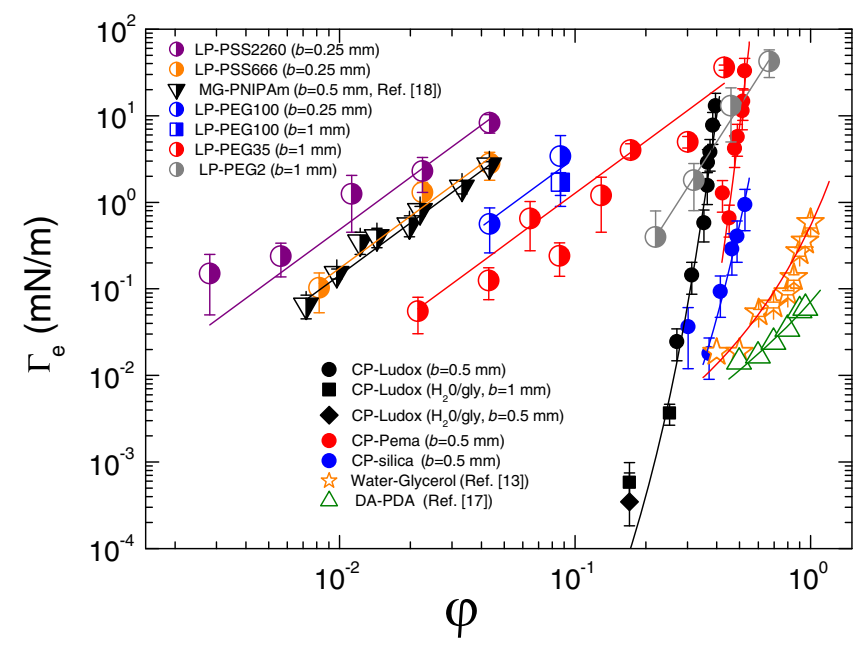

FIG. 1. Nonequilibrium interfacial tension $\Gamma_{e}$ for aqueous solutions of polymers (semifilled symbols), colloidal suspensions (full symbols), and water-glycerol and DA-PDA mixtures (empty symbols, Refs. [13] and [17], respectively). The labels indicate the gap $b$ for the Hele-Shaw experiments; the solvent is water unless specified otherwise. For all LP systems except the shortest polymer, LP-PEG2, the lines are power-law fits with an exponent 2. For all the other samples, the lines are fits of Eq. (9).

which further validates Eq. (2). For all the linear polymers except the shortest one (LP-PEG2) and for the microgels of [18], we find $\Gamma_{e} \sim \varphi^{2}$, in agreement with Korteweg's theory. For the polymer-monomer mixtures of Ref. [17], $\Gamma_{e}$ seemingly grows as $\varphi^{2}$ at the lower concentrations, but the growth becomes steeper at larger $\varphi$. For the shortest polymer (LP-PEG2), $\Gamma_{e}$ is clearly incompatible with Korteweg's law: the data may be fitted by a power law, but with a much higher exponent: $\Gamma_{e} \sim \varphi^{\beta}$ with $\beta=4.24 \pm 0.25$. The growth of $\Gamma_{e}(\varphi)$ is much steeper for the suspensions of colloidal particles: the interfacial tension of $C P$-Ludox increases by almost 5 orders of magnitude when varying $\varphi$ over a mere factor of 2; similarly steep growths of $\Gamma_{e}$ are also seen for the other colloidal suspensions. The data of Ref. [13] (up triangles) have a behavior intermediate between that of polymers and colloidal particles: the curved shape in the log-log plot of Fig. 1 rules out a power law, but the $\varphi$ dependence is less steep than for the $C P$ samples.

Not only does the particle internal structure have great influence on the functional form of $\Gamma_{e}(\varphi)$, it also significantly impacts the absolute magnitude of the interfacial tension. For the polymers that follow Korteweg's law, $\Gamma_{e}$ at fixed concentration varies by 2 orders of magnitude when changing from LP-PSS2260 to LP-PEG35. An even more spectacular change is observed between the colloidal samples and those of Refs. [13,17]: at the same $\varphi \approx 0.5$, three decades and a half separate the interfacial tension of $C P$-Pema from that of the water-glycerol or DA-PDA mixtures. Remarkably, for our complex fluids, $\Gamma_{e}$ may be as large as tens of $\mathrm{mN} \mathrm{m}^{-1}$, comparable to immiscible molecular fluids [71] and orders of magnitude larger than for colloidal phases at equilibrium, for which, typically, $\Gamma \sim 10^{-3} \mathrm{mN} \mathrm{m}^{-1}$ [72]. Note that body forces originating from these tensions dominate over gravitational forces for all particles and polymers under investigation [73].

\section{MODEL}

The square-gradient model clearly fails to capture both the variety of the functional forms and the large variability of the magnitude of $\Gamma_{e}$ shown in Fig. 1. To overcome these limitations, we present here a simple model that provides a unified framework for rationalizing the interfacial tension between miscible fluids.

Following a thermodynamic approach, the effective interfacial tension is obtained from [19]

$$
\Gamma_{e}=\frac{\partial F}{\partial A},
$$

with $F$ the mixing free energy of the two fluids and $A$ the area of the interface between them. Assuming local equilibrium [74], the free energy to be input in Eq. (3) is expressed as a sum over powers of the concentration gradient at the interface $[45,75]$ :

$$
F=\int_{V} f d V=\int_{V}\left[f_{0}+\sum_{l=1}^{\infty} \kappa_{2 l}(\tilde{\varphi})(\nabla \tilde{\varphi})^{2 l}\right] d V,
$$

where $f$ and $f_{0}$ are the mixing free-energy density of the inhomogeneous and homogeneously mixed system, respectively, and $\kappa_{2 l}(\tilde{\varphi})$ are the coefficients of the expansion. $\tilde{\varphi}$ denotes the local concentration: $\tilde{\varphi}=0(\tilde{\varphi}=\varphi)$ in the solvent (in the bulk). Note that for symmetry reasons only the even coefficients are non-null, as argued by Cahn and Hilliard [76,77]. Equation (4) extends the square-gradient model, which retains only the first term, $l=1$.

By integrating the local part of the free energy over the coordinates $(x, y)$ tangential to the interface, one obtains $F$ as an integral in $z$, the coordinate normal to the interface. By inserting this expression in Eq. (3), the desired effective interfacial tension is finally obtained as

$$
\Gamma_{e}=\int_{-\delta / 2}^{\delta / 2} d z \sum_{l=1}^{\infty} \kappa_{2 l}(\tilde{\varphi}) \frac{\varphi^{2 l}}{\delta^{2 l}} .
$$

In writing Eq. (5), we assume that the concentration gradient at the interface may be approximated by a linear profile over a thickness $\delta$ :

$$
\nabla \tilde{\varphi}=\varphi / \delta \quad-\delta / 2<z<\delta / 2
$$$$
0 \text { elsewhere. }
$$

Note that, due to diffusion, the thickness $\delta$ is an increasing function of time; it is expected to be of the order of the 
interparticle distance on time scales shorter than the time it takes a particle to diffuse over this distance.

The coefficients $\kappa_{2 l}$ in Eqs. (4) and (5) are the Taylor coefficients of the free-energy expansion around $\nabla \tilde{\varphi}=0$ :

$$
\kappa_{2 l}=\left.\frac{1}{(2 l) !} \frac{\partial^{2 l} f}{\partial(\nabla \tilde{\varphi})^{2 l}}\right|_{\nabla \tilde{\varphi}=0} .
$$

On the grounds of dimensional analysis, $\kappa_{2 l}$ is expected to scale as $\epsilon_{2 l}(\varphi) L_{2 l}^{2 l}(\varphi)$, with $\epsilon_{2 l}$ and $L_{2 l}$ (concentrationdependent) energy densities and length scales, respectively. To make progress, we assume that the energy and length scales that appear in $\kappa_{2 l}$ do not depend on either the order $l$ or on $\varphi$. We thus postulate

$$
\kappa_{2 l}=\frac{1}{(2 l) !} \epsilon L^{2 l} .
$$

Note that one expects $L \geq \delta$, since the interface thickness represents the smallest length scale in the problem. Hence, Eq. (8) implies the possibility that particles beyond the layer directly exposed to the solvent are perturbed when creating an interface. We elaborate further on this point when discussing the experimental results. With this choice, the sum on the rhs of Eq. (5) can be calculated explicitly, yielding a closed form for $\Gamma_{e}$ :

$$
\Gamma_{e}=\Gamma_{0}\left[\cosh \left(\varphi \frac{L}{\delta}\right)-1\right]
$$

where $\Gamma_{0}=\epsilon \delta$ sets the scale of the effective interfacial tension, via the energy density $\epsilon$ and the interface thickness $\delta$.

Equation (9) correctly captures the $\varphi$ dependence of all data. The Korteweg-like behavior of the polymer samples (except LP-PEG2) corresponds to the $\varphi L / \delta \lesssim 1$ regime, where Eq. (9) reduces to the quadratic scaling $\Gamma_{e} \sim \varphi^{2}$. Data for LP-PEG2 are also well reproduced, as shown by the line in Fig. 1; they correspond to the regime $1 \lesssim \varphi L / \delta \lesssim 4$, where higher-order terms start to be important and $\Gamma_{e}$ deviates sensibly from a quadratic behavior. Finally, Eq. (9) yields excellent fits to the interfacial tension for colloidal particles and the water-glycerol mixtures (lines in Fig. 1), for which $\varphi L / \delta>4$.

While Eq. (9) provides a simple yet general expression for describing the concentration dependence of $\Gamma_{e}$, it is difficult to obtain direct insight on the model parameters by fitting the experimental data: operationally, the fitting parameters are $\Gamma_{0}$ and the ratio $L / \delta$, such that $\epsilon$ and $L$ can be disentangled from the fitting parameters only by assigning a priori a value to $\delta$, e.g., $\delta$ is approximately the interparticle separation. We circumvent this difficulty by replacing $\left(\Gamma_{0} / 2\right)(\varphi L / \delta)^{2}$, the quadratic term in the Taylor expansion of the rhs of Eq. (9), by a suitable expression $\Gamma^{\mathrm{SG}}$ derived in the framework of a square-gradient approach:
$\Gamma_{e}=\Gamma^{\mathrm{SG}}(\varphi, \delta)+\Gamma_{0}\left[\cosh \left(\varphi \frac{L}{\delta}\right)-\frac{L^{2}}{2 \delta^{2}} \varphi^{2}-1\right]$.

As indicated by the notation in Eq. (10) and as we show in the following, for most systems it is possible to derive analytical expressions for $\Gamma^{\mathrm{SG}}$ that depend explicitly on $\delta$. Our generalized law for the interfacial tension, Eq. (10), can then be fitted to the data to obtain the two length scales $\delta$ and $L$ and the energy density $\epsilon$. For linear polymers and molecular liquids, $\Gamma^{\mathrm{SG}}$ can be computed using lattice theory. Here, we outline the calculations, providing all details in Ref. [54]. For polymer-solvent mixtures at equilibrium, the square-gradient coefficient $\kappa_{2}(\tilde{\varphi})$ has been computed in Ref. [78]. Its $\varphi=0$ limit has been used to compute the effective interfacial tension $\Gamma_{e}$ for microgels [18]. We generalize the calculation using the full $\tilde{\varphi}$-dependent expression of $\kappa_{2}$ and assuming a linear concentration gradient at the solvent-solution interface, finding [54]

$$
\Gamma_{p}^{\mathrm{SG}}=\frac{\kappa_{p}(\varphi)}{\delta} \varphi^{2}
$$

with

$$
\kappa_{p}(\varphi)=\frac{R T R_{g}^{2}}{6 V_{s}}\left[\chi-\frac{3}{\varphi} \ln (1-\varphi)\right]
$$

the Korteweg parameter for polymers. Here, $R$ is the ideal gas constant, $T$ the absolute temperature, $V_{s}$ the molar volume of the solvent, $R_{g}$ the gyration radius of the polymer [78], which is proportional to the hydrodynamic radius $R_{h}$ [79], and $\chi$ the Flory-Huggins parameter of the mixture $[54,63,81]$. The $\chi$ term on the rhs of Eq. (12) accounts for the enthalpic contribution to $\kappa_{p}$, while the second term in the square brackets reflects the entropic contributions. The latter term stems from the decrease of entropy due to the constraint that the chain's configuration be compatible with the concentration gradient at the interface. Crucially, this term differs from previous formulations [18] by the $\varphi^{-1} \ln (1-\varphi)$ factor, which we show to be necessary to correctly account for some of the polymer data. Note that all quantity defining $\kappa_{p}$ are known or independently measurable: the only unknown in the rhs of Eq. (11) is thus the interface thickness $\delta$, which will be treated as a fitting parameter.

To the best of our knowledge, no explicit expressions have been derived for $\kappa_{f}$, the Korteweg parameter for miscible molecular fluids. We calculate both the enthalpic and the entropic contributions to $\kappa_{f}$ using lattice theory arguments and assuming for simplicity that the mixture is symmetric, i.e., that the two fluids have the same molecular volume $\Omega$. The two terms are due, respectively, to the variation of the internal energy density $u$ and the decrease of configurational entropy density $s$ in the region where 
$|\nabla \varphi|>0$. They are obtained by expressing $u$ and $s$ as a function of the local concentration, assuming a concentration gradient across three adjacent lattice layers orthogonal to the $z$ direction, and finally by expanding the local concentration around that of the central layer, up to second order in the spatial derivatives of $\tilde{\varphi}$. As detailed in Ref. [54], we find

$$
\Gamma_{f}^{\mathrm{SG}}=\frac{\kappa_{f}(\varphi)}{\delta} \varphi^{2}
$$

where the Korteweg parameter for molecular fluids is

$\kappa_{f}(\varphi)=\frac{k_{b} T a^{2}}{\Omega}\left\{\frac{\chi}{6}+\frac{2}{3 \varphi^{2}}\left[1+\frac{1-\varphi}{\varphi} \ln (1-\varphi)\right]\right\}$,

with $k_{b}$ Boltzman's constant and $a$ the radius of the fluid molecules. The first term on the rhs of Eq. (14), proportional to the $\chi$ parameter that characterizes the interaction between the small molecules of the two fluids, quantifies the energy penalty (or gain) due to a local compositional inhomogeneity, while the second term, always positive and only dependent on $\varphi$, is due to the entropy loss due to the (transient) gradient of concentration. As a final remark on the expressions for $\Gamma^{\mathrm{SG}}$ for polymers and molecular fluids, we note that the approximations used to derive Eqs. (11)-(14) imply $\varphi \neq 0, \varphi \neq 1$ and that the concentration gradient at the interface be small, i.e., that the effective interfacial tension be dominated by $\Gamma^{\mathrm{SG}}$.

\section{DATA ANALYSIS AND DISCUSSION}

We now use the general expression for the interfacial tension, Eq. (10), together with Eqs. (11) and (13), to model the data shown in Fig. (1). The data for the microgels and all linear polymers, except the shortest chain LP-PEG2, are well accounted for by the square-gradient expression for polymers with no need to add higher-order terms, i.e., the term proportional to $\Gamma_{0}$ in Eq. (10). We thus use Eq. (11) with Eq. (12), where the only fit parameter is the interface thickness $\delta$. The fit quality is excellent over 2 decades in $\varphi$, as shown in Fig. 2(a), where we plot the interfacial tension in reduced units, $\delta \Gamma_{e} / \kappa_{p}$. The inset shows the fit parameter $\delta$ as a function of the radius of gyration of the polymers and microgels: we find that the interface thickness is proportional to the polymer size, $\delta=2 m R_{g}$, with a proportionality coefficient $m=2.2 \pm 0.2$ of order unity. This is consistent with the fact that our experiments are performed fast enough for interdiffusion between the polymer solution and the injected solvent to be negligible, a regime where the interface thickness is expected to be comparable to the interparticle distance, which in turn is close to the particle size [82]. The $\delta \sim R_{g}$ scaling, together with the size dependence of $\kappa_{p}$, Eq. (12), implies $\Gamma_{e} \sim R_{g}$, consistent
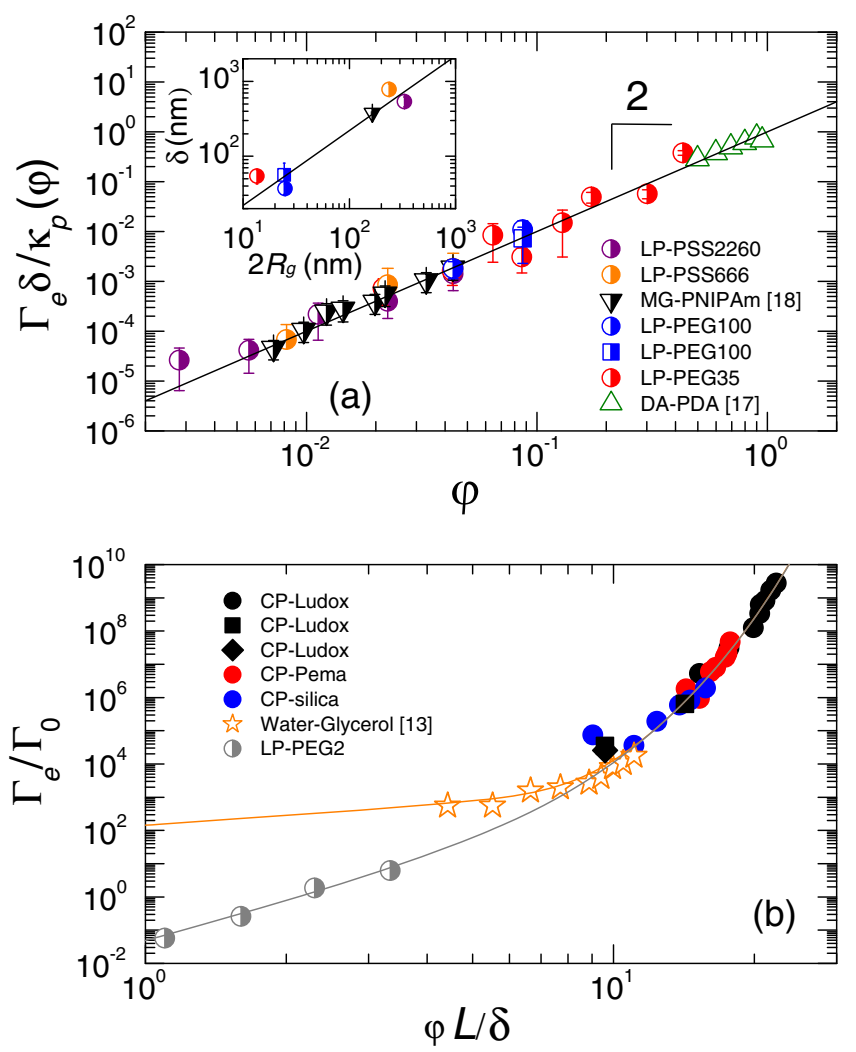

FIG. 2. Nonequilibrium interface tension (same data as in Fig. 1), plotted using the reduced variables suggested by Eqs. (10), (11), and (13). (a) Data for all polymer systems except LP-PEG2. The line is the law $\Gamma_{e} \delta / \kappa_{p}(\varphi)=\varphi^{2}$ predicted by the theory. Inset: Interface thickness obtained from the fits as a function of the polymer size. The line is the linear law $\delta=4.4 R_{g}$. (b) Normalized interfacial tension for colloidal suspensions, short polymers (LP-PEG2), and water-glycerol mixtures. The lines are fits of Eq. (10), with $\Gamma^{\mathrm{SG}}=0$ for $C P$ suspensions and $\Gamma^{\mathrm{SG}}$ as in Eqs. (11) and (13) for LP-PEG2 and water-glycerol mixtures, respectively.

with the growth of $\Gamma_{e}$ with the degree of polymerization seen in Fig. 1 for the PEG-based samples.

Quite remarkably, Eqs. (11) and (12) also capture very well the spinning drop tensiometry data for DA-PDA mixtures [17]. The authors of Ref. [17] explicitly discuss the failure of a simple quadratic scaling $\Gamma_{e} \sim \varphi^{2}$ for their data, which is clearly seen in a log-log graph (see the upward curvature of the up triangles' data set in Fig. 1). By contrast, the excellent quality of the fit shown in Fig. 2(a) demonstrates that a more-refined formulation of the squaregradient theory is, in fact, adequate to model the data, in spite of the large compositional mismatch between the two fluids. The success of the square-gradient-based model, Eq. (11), is due to the small concentration gradient, because of the diffusion-driven smearing of the interface on the time scale of the experiment: the fit yields $\delta=68 \pm$ $20 \mu \mathrm{m}$ [83], more than $10^{4}$ times the molecular size, and is consistent with $\delta \approx 10^{2} \mu \mathrm{m}$, as estimated by optical means in Ref. [17]. 
For the shortest polymer, LP-PEG2, the water-glycerol mixtures and the colloidal particle systems, neither the basic Korteweg law, Eq. (1), nor its refined versions, Eqs. (11) and (13), are adequate to model the data. We thus use the full expression, Eq. (10), finding in all cases an excellent agreement between the fit and the data, as seen in Fig. 2(b). For LP-PEG2, $\Gamma^{\mathrm{SG}}$ is evaluated using Eqs. (11) and (12): the fitting parameters are thus $\delta, L$, and $\epsilon$. We find $\delta=10 \pm 8 \mathrm{~nm}$, somehow larger than $\delta \approx 6.8 \mathrm{~nm}$, as estimated from the $\delta$ versus $R_{g}$ scaling established for the other polymers (inset of Fig. 1). This slight discrepancy is likely due to the fact that on the time scale of the experiment interdiffusion is not fully negligible for LP-PEG2, leading to a slight smearing of the interface [55]. We discuss the values of the other fitting parameters in the following, together with those for the other sets of data of Fig. 2(b).

We fit the data for water-glycerol mixtures of Ref. [13] using Eq. (10) with $\Gamma^{\mathrm{SG}}$ given by Eqs. (13) and (14); the fitting parameters are again $\delta, L$, and $\epsilon$. The interface thickness obtained from the fit is $\delta=66 \pm 30 \mathrm{~nm}$, much larger than the molecular size, as expected due to the high diffusivity of molecular fluids, but still fully compatible with an upper bound of about $0.2 \mu \mathrm{m}$, set by the remark that no interdiffusion could be detected by optical observations, as pointed out in Ref. [13].

For the colloidal suspensions, no analytical expression is available for $\Gamma^{\mathrm{SG}}$. Furthermore, $\Gamma_{e}$ is dominated by the higher-order terms of the development in powers of the concentration gradient: by testing various trial values of $\Gamma^{\mathrm{SG}}$, we find that the fit quality does not depend appreciably on the square-gradient term, as long as $\left|\Gamma^{\mathrm{SG}}\right| \lesssim 10^{4} \Gamma_{0}$. We conclude that, within experimental uncertainties, the second term of the rhs of Eq. (10) is sufficient to reproduce very well the $C P$ experiments, and that the data are compatible with a non-null, albeit very small, value of $\Gamma^{\mathrm{SG}}$, including the possibility that the contribution of the square-gradient term be negative $[42,54]$. For the sake of simplicity, we set $\Gamma^{\mathrm{SG}}=0$ in Eq. (10) and fit the $C P$ data with $\Gamma_{0}$ and $L / \delta$ as fitting parameters.

For all systems for which the square-gradient model is not sufficient to describe $\Gamma_{e}$, the fitting parameters $L / \delta$ and $\Gamma_{0}$ may be used to build master curves. As seen in Fig. 2(b), two regimes may be identified when plotting $\Gamma_{e} / \Gamma_{0}$ versus the scaled concentration $\varphi L / \delta$. For $\varphi L / \delta \gtrsim 10$, the contribution of $\Gamma^{\mathrm{SG}}$ is negligible: this is the case of the $C P$ suspensions, for which all data collapse on a master curve. The high- $\varphi$ extrapolation of the fits for LP-PEG2 and water-glycerol [lines in Fig. 2(b)] also belong to this regime and fall onto the same master curve. By contrast, at lower $\varphi L / \delta$ the contribution of the square-gradient term becomes important. Since the relative weight of $\Gamma^{\mathrm{SG}}$ with respect to $\Gamma_{0}$ is system dependent, the fits to LP-PEG2 and to the data of Ref. [13] form distinct branches of the master curve of Fig. 2(b). In particular, the empirical power-law behavior $\Gamma_{e} \sim \varphi^{4.24}$ for LP-PEG2 (see also Fig. 1) is, in fact, a crossover between the exponential growth of $\Gamma_{e}$ at large $\varphi L / \delta$ and the $\varphi^{2}$ behavior expected in the limit $\varphi \rightarrow 0$.

Figure 3 shows the ratio $L / \delta$ for the five systems of Fig. 2(b). In all cases, $L>\delta$. Since the interface thickness $\delta$ is of the order of the interparticle spacing, the largest structural length scale for a system at equilibrium, we argue that the emergence of a larger length scale $L$ is a consequence of the system being strongly driven. Longrange spatial correlations, up to more than 1 order of magnitude larger than the equilibrium structural length scale, have been observed in the structure and the dynamics of a variety of driven systems, from flowing granular matter [84,85] to colloidal [86,87] and polymer systems [88] where the Péclet number, the ratio of the Brownian diffusion time over the advection time imposed by the drive, is larger than unity. To quantify the importance of the drive in our Hele-Shaw geometry, we calculate the Péclet number $\mathrm{Pe}=\left(2 R_{h}\right)^{3} \pi \eta_{2} \dot{\gamma}_{r} /\left(k_{B} T\right)$. For the polymer and colloid suspensions reported in Fig. 3, we find that Pe is larger than unity and ranges from 2.3 to 680 , supporting the notion that the additional length scale $L$ stems from the system exploring microscopic configurations different from the equilibrium ones.

Interestingly, the trend of the data for the $C P$ samples reflects the difference in the range of particle interactions, $L / \delta$ being smallest for $C P$-silica, for which the interactions are close to hard sphere and thus shorter ranged as compared to those for $C P$-Pema and $C P$-Ludox. Both the water-glycerol mixtures and the short polymer LPPEG2 have a $L / \delta$ ratio significantly smaller than that of colloidal suspensions. We attribute this effect to a gentler concentration profile at the suspension-solvent interface. Indeed, we recall that all the experiments involving $C P$ have been performed on a time scale shorter than the diffusion-driven relaxation time of the interface [55]. Under these conditions, one expects $\delta \sim 2 R_{h}$. By contrast, for water-glycerol and LP-PEG2, $\delta$ is estimated to be larger than the polymer or molecule size, due to diffusion-driven smearing of the interface. Accordingly, the concentration

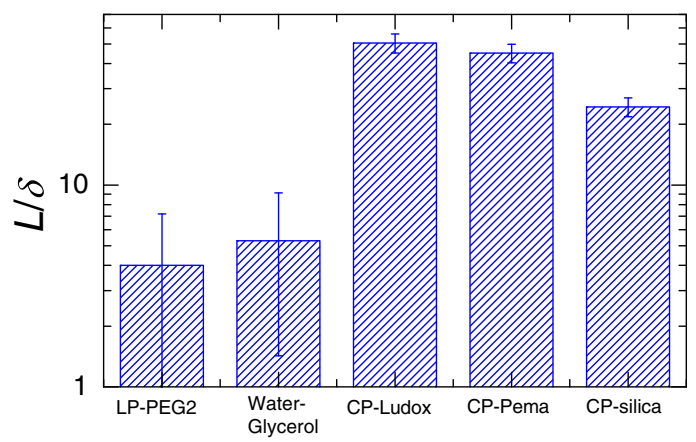

FIG. 3. Ratio $L / \delta$ obtained by fitting the data shown in Fig. 2(b) with Eq. (10). Large values of $L / \delta$ are obtained for $C P$ samples where interfaces are probed on a time scale shorter than the diffusion-driven relaxation time of the interface. 
gradient is smaller than for the $C P$ systems, the configuration of the layers adjacent to the interface is less perturbed, and the interfacial tension behavior is closer to its square-gradient limit, corresponding to smaller $L / \delta$ ratios.

The energy density $\epsilon$ obtained by fitting Eq. (10) to the data of Fig. 2(b) is shown in Table III. Remarkably, $\epsilon$ exhibits a very strong system dependence, ranging from $\sim 10^{-5}$ Pa for colloidal Pema to more than $\sim 10^{6} \mathrm{~Pa}$ for the short polymer LP-PEG2. In order to rationalize such a wide range of values, we consider the LP-PEG2 and $C P$-silica samples, which are both characterized by excluded-volume interactions, with no enthalpic or Coulombic contributions as for the other samples, and for which $\Gamma_{e}$ is measured in the $t \rightarrow 0$ limit, unlike for the water-glycerol mixtures. Under these conditions, we expect the effective interfacial tension to have a purely entropic origin and $\epsilon$ to be the freeenergy density associated to the frustration of degrees of freedom due to the additional constraint of creating a concentration gradient at the interface. For $C P$-silica, we estimate the cost in energy density due to excluded volume interactions as the thermal energy $k_{b} T$ divided by the particle volume $\left(2 R_{h}\right)^{3}$, yielding $\epsilon=1.49 \times 10^{-3} \mathrm{~Pa}$, close to the energy density obtained by fitting our model to the data. Similarly, for a LP-PEG2 chain the energy cost due to excluded volume interactions is estimated as the thermal energy $k_{b} T$ times the number $N$ of monomers in the chain. The energy density is thus $\epsilon \sim k_{b} T N / R_{F}^{3}$, where $R_{F}=$ $b_{m} N^{0.58}$ is the coil size in a good solvent and $b_{m}$ the monomer length. Using $b_{m}=0.35 \mathrm{~nm}$ and $N=32$, we obtain $\epsilon=7.26 \times 10^{6} \mathrm{~Pa}$, again of the same order of magnitude as the value obtained from the fit. As a further consistency check, we also evaluate $\epsilon$ for the longer PEG polymers, by rescaling the result for LP-PEG2 to take into account the difference in the polymerization degree $N$. We find $\epsilon=1.68 \times 10^{5} \mathrm{~Pa}$ for LP-PEG35 $\left(\epsilon=7.70 \times 10^{4} \mathrm{~Pa}\right.$ for LP-PEG100); with these values, we calculate the relative contribution to $\Gamma_{e}$ stemming from the terms with powers of $\varphi$ larger than 2 to be smaller than $10 \%$ for LPPEG35 and as low as $3.4 \times 10^{-4}$ for LP-PEG100. Therefore, our model explains quantitatively why the square-gradient theory is sufficient to reproduce the concentration dependence of the interfacial tension for these samples, while an anomalous $\Gamma_{e} \sim \varphi^{4.2}$ scaling is observed for the shorter polymer LP-PEG2.

TABLE III. Energy density $\epsilon$ and its relative uncertainty $\Delta \epsilon$ as obtained from the fits to the data shown in Fig. 2(b).

\begin{tabular}{lcc}
\hline \hline Code & $\epsilon(\mathrm{Pa})$ & $\Delta \epsilon(\%)$ \\
\hline LP-PEG2 & $1.4 \times 10^{6}$ & 86 \\
Water-glycerol & 0.5 & 60 \\
CP-Ludox & $9.2 \times 10^{-4}$ & 130 \\
CP-Pema & $9.9 \times 10^{-6}$ & 131 \\
CP-silica & $3.2 \times 10^{-3}$ & 131 \\
\hline \hline
\end{tabular}

Our experiments have shed new light on the interfacial tension between miscible fluids, unveiling surprisingly large variations of $\Gamma_{e}$ according to the fluid composition, and revealing a concentration dependence much stronger than in earlier reports. Korteweg's century-old squaregradient theory cannot capture this fascinating richness of behavior. The simple model we introduce here is a first step towards a rationalization of these results. The model extends Korteweg's approach by introducing a length and an energy density scale, whose magnitude are consistent with estimates based on simple physical arguments; in spite of its simplicity, it reproduces remarkably well both our new measurements and existing literature data on molecular and polymer fluids. Further work is needed to achieve a finer understanding of interfacial stresses in miscible fluids: open questions concern, in particular, the role of the interparticle potential, the time behavior of the interfacial tension, and the possible existence of a transiently negative interfacial tension in the dilute regime.

At a more general level, we expect the model we develop here to be applicable to other systems where the presence of an interface between different phases plays a key role. Density-functional theory and phase field methods have been used to tackle problems of this kind in the context of phase transitions, metallurgy, and material science. Both approaches typically rely on a square-gradient description of the interface contribution: such an approximation is now recognized as a crucial limitation preventing a more faithful modeling, especially for sharp interfaces [44,47-51]. Current strategies to overcome the square-gradient approach are based on the inclusion of a quartic gradient term, the next term in a Taylor expansion of $F$ [48-50]. By providing an analytical form for the full contribution of the interface to the free energy, our model represents a significant advance with respect to low-order Taylor expansions.

\section{ACKNOWLEDGMENTS}

This work has been supported by the French National Research Agency (ANR) (Grants No. ANR-2010-BLAN0402-1, F2F, No. ANR-14-CE32-0005-01, FAPRES). The authors are grateful to J.-L. Barrat, M. Bier, P. Petitjeans, and J. A. Pojman for useful discussions.

[1] J.D. van der Waals, The Thermodynamic Theory of Capillarity under the Hypothesis of a Continuous Variation of Density, Z. Phys. Chem. 13, 657 (1894).

[2] D. M. Anderson, G. B. McFadden, and A. A. Wheeler, Diffuse-Interface Methods in Fluid Mechanics, Annu. Rev. Fluid Mech. 30, 139 (1998).

[3] J. Rowlinson and B. Widom, Molecular Theory of Capillarity (Clarendon, Oxford, 1982). 
[4] P.-G. de Gennes, F. Brochard-Wyart, and D. Queré, Capillarity and Wetting Phenomena: Drops, Bubbles, Pearls, Waves (Springer-Verlag, Berlin, 2004).

[5] S. Mora, T. Phou, J.-M. Fromental, L. M. Pismen, and Y. Pomeau, Capillarity Driven Instability of a Soft Solid, Phys. Rev. Lett. 105, 214301 (2010).

[6] A. Marchand, S. Das, J. H. Snoeijer, and B. Andreotti, Capillary Pressure and Contact Line Force on a Soft Solid, Phys. Rev. Lett. 108, 094301 (2012).

[7] L. Bocquet and E. Charlaix, Nanofluidics, from Bulk to Interfaces, Chem. Soc. Rev. 39, 1073 (2010).

[8] L. Bocquet and P. Tabeling, Physics and Technological Aspects of Nanofluidics, Lab Chip 14, 3143 (2014).

[9] D. J. Korteweg, Sur la Forme que Prennent les Équations du Mouvement des Fluides si l'on Tient Compte des Forces Capillaires Causées par des Variations de Densité Considérables Mais Continues et sur la Théorie de la Capillarité dans l'hypothèse d'une Variation Continue de la Densité, Arch. Neerland. Sci. Exactes Naturelles 6, 1 (1901).

[10] H. T. Davis, Numerical Simulation and Oil Recovery (Springer-Verlag, Berlin, 1988), p. 105.

[11] Y. B. Zeldovich, About Surface Tension of a Boundary between Two Mutually Soluble Liquids, Zh. Fi. Khim. 23, 931 (1949).

[12] P. G. Smith, T. G. M. Van den Ven, and S. G. Mason, Transient Interfacial Tension between Two Miscible Liquids, J. Colloid Interface Sci. 80, 302 (1981).

[13] P. Petitjeans, Une Tension de Surface pour les Fluides Miscibles, C. R. Acad. Sci. Paris 322, 673 (1996).

[14] P. Cicuta, A. Vailati, and M. Giglio, Capillary-to-Bulk Crossover of Nonequilibrium Fluctuations in the Free Diffusion of a Near-Critical Binary Liquid Mixture, Appl. Opt. 40, 4140 (2001).

[15] L. Lacaze, P. Guenoun, D. Beysens, M. Delsanti, P. Petitjeans, and P. Kurowski, Transient Surface Tension in Miscible Liquids, Phys. Rev. E 82, 041606 (2010).

[16] J. A. Pojman, C. Whitmore, M. L. Turco Liveri, R, Lombardo, J. Marszalek, R. Parker, and B. Zoltowski, Evidence for the Existence of an Effective Interfacial Tension between Miscible Fluids: Isobutyric Acid-Water and 1-Butanol-Water in a Spinning-Drop Tensiometer, Langmuir 22, 2569 (2006).

[17] B. Zoltowski, Y. Chekanov, J. Masere, J. A. Pojman, and V. Volpert, Evidence for the Existence of an Effective Interfacial Tension between Miscible fluids. 2. Dodecyl Acrylate-Poly(Dodecyl Acrylate) in a Spinning Drop Tensiometer, Langmuir 23, 5522 (2007).

[18] D. Truzzolillo, S. Mora, C. Dupas, and L. Cipelletti, OffEquilibrium Surface Tension in Colloidal Suspensions, Phys. Rev. Lett. 112, 128303 (2014).

[19] D. Truzzolillo and L. Cipelletti, Off-Equilibrium Surface Tension in Miscible Fluids, Soft Matter, doi: 10.1039/ c6sm01026a (2016).

[20] G. Morra and D. A. Yuen, Role of Korteweg Stresses in Geodynamics, Geophys. Res. Lett. 35, n/a (2008).

[21] A. Souriau and G. Poupinet, A Study of the Outermost Liquid Core Using Differential Travel Times of the SKS, SKKS and S3KS Phases, Phys. Earth Planet. Inter. 68, 183 (1991).
[22] A. De Wit, Y. Bertho, and M. Martin, Viscous Fingering of Miscible Slices, Phys. Fluids 17, 054114 (2005).

[23] S. Swernath, B. Malengier, and S. Pushpavanam, Effect of Korteweg Stress on Viscous Fingering of Solute Plugs in a Porous Medium, Chem. Eng. Sci. 65, 2284 (2010).

[24] P. Petitjeans and T. Maxworthy, Miscible Displacements in Capillary Tubes. Part 1. Experiments, J. Fluid Mech. 326, 37 (1996).

[25] J. W. Jawitz, M. D. Annable, and P. S. C. Rao, Miscible Fluid Displacement Stability in Unconfined Porous Media: Two-Dimensional Flow Experiments and Simulations, J. Contam. Hydrol. 31, 211 (1998).

[26] T. Babadagli, Development of Mature Oil Fields-A Review., J. Pet. Sci. Eng. 57, 221 (2007).

[27] X. Liu, E. George, W. Bo, and J. Glimm, Turbulent Mixing with Physical Mass Diffusion, Phys. Rev. E 73, 056301 (2006).

[28] C.-Y Chen, C.-W Huang, H. Gadêlha, and J. A. Miranda, Radial Viscous Fingering in Miscible Hele-Shaw Flows: A Numerical Study, Phys. Rev. E 78, 016306 (2008).

[29] E. O. Dias and J. A. Miranda, Wavelength Selection in HeleShaw Flows: A Maximum-Amplitude Criterion, Phys. Rev. E 88, 013016 (2013).

[30] T. Ban, A. Aoyama, and T. Matsumoto, Self-Generated Motion of Droplets Induced by Korteweg Force, Chem. Lett. 39, 1294 (2010).

[31] T. Ban, T. Fukuyama, S. Makino, E. Nawa, and Y. Nagatsu, Self-Propelled Vesicles Induced by the Mixing of Two Polymeric Aqueous Solutions through a Vesicle Membrane Far from Equilibrium, Langmuir 32, 2574 (2016).

[32] N. K. Adam, The Physics and Chemistry of Surfaces (Dover, New York, 1968).

[33] L. Paterson, Fingering with Miscible Fluids in a Hele Shaw Cell, Phys. Fluids 28, 26 (1985).

[34] J. Nittmann, G. Daccord, and H. E. Stanley, Fractal Growth Viscous Fingers: Quantitative Characterization of a Fluid Instability Phenomenon, Nature (London) 314, 141 (1985).

[35] J. Nittmann and H. E. Stanley, Tip Splitting without Interfacial Tension and Dendritic Growth Patterns Arising from Molecular Anisotropy, Nature (London) 321, 663 (1986).

[36] S. E. May and J. V. Maher, Capillary-Wave Relaxation for a Meniscus between Miscible Liquids, Phys. Rev. Lett. 67, 2013 (1991).

[37] D. D. Joseph, Fluid Dynamics of Two Miscible Liquids with Diffusion and Gradient Stresses, Eur. J. Mech. B 9, 565 (1990).

[38] J. E. Mungall, Interfacial Tension in Miscible Two-Fluid Systems with Linear Viscoelastic Rheology, Phys. Rev. Lett. 73, 288 (1994).

[39] P. Garik, J. Hetrick, B. Orr, D. Barkey, and E. Ben-Jacob, Interfacial Cellular Mixing and a Conjecture on Global Deposit Morphology, Phys. Rev. Lett. 66, 1606 (1991).

[40] A. Castellanos and A. González, Interfacial Electrohydrodynamic Instability: The Kath and Hoburg Model Revisited, Phys. Fluids A 4, 1307 (1992).

[41] D. Truzzolillo, V. Roger, C. Dupas, S. Mora, and L. Cipelletti, Bulk and Interfacial Stresses in Suspensions of Soft and Hard Colloids, J. Phys. Condens. Matter 27, 194103 (2015). 
[42] M. Bier, Nonequilibrium Interfacial Tension during Relaxation, Phys. Rev. E 92, 042128 (2015).

[43] T. Speck, Stochastic Thermodynamics for Active Matter, Europhys. Lett. 114, 30006 (2016).

[44] L. T. Kong, D. Vriesinga, and C. Denniston, Quantitative Results for Square Gradient Models of Fluids, Europhys. Lett. 93, 50004 (2011).

[45] R. Evans, Density Functional Theory for Inhomogeneous Fluids I: Simple Fluids in Equilibrium, in Proceedings of the 3rd Warsaw School of Statistical Physics, Kazimierz Dolny, 2009, http://agenda.albanova.se/getFile.py/access? contribId $=260 \&$ res $I d=251 \&$ material $\mathrm{Id}=250 \&$ confId $=2509$.

[46] N. Provatas and K. Elder, Phase-Field Methods in Materials Science and Engineering (Wiley-VCH, Weinheim, 2010).

[47] K. R. Elder, M. Grant, N. Provatas, and J. M. Kosterlitz, Sharp Interface Limits of Phase-Field Models, Phys. Rev. E 64, 021604 (2001).

[48] Y. Wang and J. Li, Phase Field Modeling of Defects and Deformation, Acta Mater. 58, 1212 (2010).

[49] F. Abdeljawad, D. L. Medlin, J. A. Zimmerman, K. Hattar, and S.M. Foiles, A Diffuse Interface Model of Grain Boundary Faceting, J. Appl. Phys. 119, 235306 (2016).

[50] M. J. Borden, T. J. R. Hughes, C. M. Landis, and C. V. Verhoosel, A Higher-Order Phase-Field Model for Brittle Fracture: Formulation and Analysis within the Isogeometric Analysis Framework, Comput. Methods Appl. Mech. Eng. 273, 100 (2014).

[51] M. Plapp, Remarks on Some Open Problems in Phase-Field Modelling of Solidification, Philos. Mag. 91, 25 (2011).

[52] N. Maleki-Jirsaraei, A. Lindner, S. Rouhani, and D. Bonn, Saffman-Taylor Instability in Yield Stress Fluids., J. Phys. Condens. Matter 17, S1219 (2005).

[53] A. Lindner, D. Bonn, and J. Meunier, Phys. Fluids 12, 256 (2000).

[54] See Supplemental Material at http://link.aps.org/ supplemental/10.1103/PhysRevX.6.041057 for calculation of the square gradient coefficient for polymer-solvent mixtures, simple liquids, and hard sphere suspensions, calculation of the interaction parameter $\chi$ for water-glycerol mixtures, measurement of the shear-rate-dependent viscosity of polymer and colloidal suspensions, selected patterns observed in Hele-Shaw experiments, and the procedure to measure the surface tension $\Gamma_{e}$ and the number $n_{A}$ of fingers and its uncertainty.

[55] For LP-PEG2 dissolved in water at $\varphi=0.25$, for which diffusivity is highest, the longest injection time is $t_{\exp }=5 \mathrm{~ms}$, to be compared to $0.1 \mathrm{~ms}$, the time it takes a chain to diffuse over its own diameter.

[56] J. Bear, Dynamics of Fluids in Porous Media (Dover Publication, Inc., New York, 1972).

[57] The LP-PSS are charged polymers, whose conformational state (rod- or coil-like) depends on the polymer molecular weight and the ionic strength $I$ of the solution. For our saltfree LP-PSS solutions, $I=0.5 \beta C_{p}$, where $\beta=0.36$ is the fraction of uncondensed counterions evaluated according to the Manning condensation theory [58] and $C_{p}$ is the polymer concentration in monomolar. For the lowest LPPSS concentration investigated $\left(\varphi=2.8 \times 10^{-3}=10.8\right.$ monomol), $I=1.94 \mathrm{mM}$, resulting in a persistence length
$L_{p} \approx 10 \mathrm{~nm}$ [59], much smaller than the hydrodynamic diameter $2 R_{h}=158 \mathrm{~nm}(220 \mathrm{~nm})$ of LP-PSS666 (LPPSS2260). At higher $\varphi, I$ increases, resulting in an even lower $L_{p}$. This shows that the LP-PSS polymers are far from a rodlike configuration (for which $L_{p} \gg 2 R_{h}$ ), justifying that the same arguments used to model $\Gamma_{e}$ for the uncharged, coil-like polymers will be used for the LP-PSS solutions.

[58] G. S. Manning, Limiting Laws and Counterion Condensation in Polyelectrolyte Solutions II. Self-Diffusion of the Small Ions, J. Chem. Phys. 51, 934 (1969).

[59] V. Degiorgio, F. Mantegazza, and R. Piazza, Transient Electric Birefringence Measurement of the Persistence Length of Sodium Polystyrene Sulfonate, Europhys. Lett. 15, 75 (1991).

[60] H. Senff and W. Richtering, Temperature Sensitive Microgel Suspensions: Colloidal Phase Behavior and Rheology of Soft Spheres, J. Chem. Phys. 111, 1705 (1999).

[61] W. Stöber, A. Fink, and E. Bohn, Controlled Growth of Monodisperse Silica Spheres in the Micron Size Range, J. Colloid Interface Sci. 26, 62 (1968).

[62] E. Unzueta and J. Forcada, Semicontinuous Emulsion Copolymerization of Methyl-Methacrylate and n-Butyl Acrylate. 2. Effect of Mixed Emulsifier in Unseeded Polymerization, Polymer 36, 4301 (1995).

[63] A. Eliassi, H. Modarress, and G. A. Mansoori, Measurement of Activity of Water in Aqueous Poly(Ethylene Glycol) Solutions (Effect of Excess Volume on the Flory-HugginsParameter), J. Chem. Eng. Data 44, 52 (1999).

[64] V. M. Prabhu, M. Muthukumar, G. D. Wignall, and Y. B. Melnichenko, Dimensions of Polyelectrolyte Chains and Concentration Fluctuations in Semidilute Solutions of Sodium-Poly(Styrene Sulfonate) as Measured by SmallAngle Neutron Scattering, J. Polym. 42, 8935 (2001).

[65] Microgel Suspensions: Fundamentals and Applications, edited by A Fernandez-Nieves, H. Wyss, J. Mattsson, and D. A. Weitz (Wiley-VCH Verlag GmbH and Co., Weinheim, 2011).

[66] Irvin M. Krieger, Rheology of Monodisperse Latices, Adv. Colloid Interface Sci. 3, 111 (1972).

[67] A. Holmberg, L. Piculell, P. Schurtenberger, and U. Olsson, Oil-Continuous Microemulsions Mixed with an Amphiphilic Graft Copolymer or with the Parent Homopolymer PolymerDroplet Interactions as Revealed by Phase Behavior and Light Scattering, Colloids Surf. A 250, 325 (2004).

[68] P. J. Flory, Principles of Polymer Chemistry (Cornell University Press, Ithaca, 1953).

[69] D. D. Joseph and J. C. Saut, Short-Wave Instabilities and IllPosed Initial-Value Problems, Theor. Comput. Fluid. Dynamics. 1, 191 (1990).

[70] H. H. Hu and D. D. Joseph, Miscible Displacement in a Hele-Shaw Cell, ZAMP 43, 626 (1992).

[71] As an example, $\Gamma \approx 40 \mathrm{mN} / \mathrm{m}$ for water-silicon oil [18].

[72] E. H. A. de Hoog and H. N. W. Lekkerkerker, Measurement of the Interfacial Tension of a Phase-Separated ColloidPolymer Suspension, J. Phys. Chem. B 103, 5274 (1999).

[73] For the less concentrated suspension of the most dense and big particles ( $C P$-silica), the ratio between the body force $F_{e}$ originating from effective interfacial tension and the gravitational force $F_{g}$ is $F_{e} / F_{g}=\left[\left(2 \Gamma_{e} R_{h}\right) /(\Delta \rho g V)\right]=$ $1.73 \times 10^{8}$, where $\Delta \rho=10^{3} \mathrm{~kg} / \mathrm{m}^{3}$ is the density mismatch between particles and solvent (water), $g$ the acceleration due to gravity, and $V=4 / 3 \pi R_{h}^{3}$ the volume of one colloid. 
[74] S. Kjelstrup and D. Bedeaux, Non-Equilibrium Thermodynamics of Heterogeneous Systems (World Scientific Publishing Co. Pte. Ltd., Singapore, 2008).

[75] R. Mauri, Non-Equilibrium Thermodynamics in Multiphase Flows (Springer, Dordrecht, 2008).

[76] J. W. Cahn and J. E. Hilliard, Free Energy of a Nonuniform System. I. Interfacial Free Energy, J. Chem. Phys. 28, 258 (1958).

[77] D. A. Cogswell and W. C. Carter, Thermodynamic PhaseField Model for Microstructure with Multiple Components and Phases: The Possibility of Metastable Phases, Phys. Rev. E 83, 061602 (2011).

[78] N. P. Balsara and E. B. Nauman, The Entropy of Inhomogeneous Polymer-Solvent Systems, J. Polym. Sci., Part B: Polym. Phys. 26, 1077 (1988).

[79] The gyration radius of the linear polymers has been calculated knowing that for real polymers in good solvent $R_{h} / R_{g}=0.640$ [80] and for microgels in water $R_{g} \simeq 0.5 R_{h}$ [18]. For water-glycerol mixtures, the molecular size has been set equal to the one of glycerol.

[80] I. Teraoka, Polymer Solutions. An Introduction to Physical Properties (John Wiley and Sons, Inc. Publication, New York, 2002).

[81] V. M. Prabhu, M. Muthukumar, G. D. Wignall, and Y. B. Melnichenko, Dimensions of Polyelectrolyte Chains and
Concentration Fluctuations in Semidilute Solutions of Sodium-Poly(Styrene Sulfonate) as Measured by SmallAngle Neutron Scattering, Polymer 42, 8935 (2001).

[82] For all our polymer samples, $\varphi \gtrsim \varphi^{*}$ (see Table I), such that the distance between polymers is comparable to the polymer size and $\delta \sim R_{g}$. At lower $\varphi$, one would rather expect $\delta$ to be on the order of the distance between polymers.

[83] We evaluate $\kappa_{p}$ using $V_{s}=271.9 \times 10^{-6} \mathrm{~m}^{3} \mathrm{~mol}^{-1}$, calculated from the molecular weight of DA $\left(240 \mathrm{~g} \mathrm{~mol}^{-1}\right)$ and its mass density, $\rho=884 \mathrm{~kg} \mathrm{~m}^{-3}$.

[84] J. A. G. Orza, R. Brito, T. P. C. Van Noije, and M. H. Ernst, Patterns and long-range correlations in idealized granular flows, Int. J. Mod. Phys. C 08, 953 (1997).

[85] H. Katsuragi, A. R. Abate, and D. J. Durian, Jamming and Growth of Dynamical Heterogeneities versus Depth for Granular Heap Flow, Soft Matter 6, 3023 (2010).

[86] E. Guazzelli and J. Hinch, Fluctuations and Instability in Sedimentation, Annu. Rev. Fluid Mech. 43, 97 (2011).

[87] V. Chikkadi, G. Wegdam, D. Bonn, B. Nienhuis, and P. Schall, Long-Range Strain Correlations in Sheared Colloidal Glasses, Phys. Rev. Lett. 107, 198303 (2011).

[88] X.-L. Wu, D. J. Pine, and P. K. Dixon, Enhanced Concentration Fluctuations in Polymer Solutions under Shear Flow, Phys. Rev. Lett. 66, 2408 (1991). 